Int. J. Dev. Biol. 55: 399-405

doi: $10.1387 / \mathrm{ijdb} .103218 \mathrm{ap}$

\title{
Modeling of angioadaptation: insights for vascular development
}

\author{
AXEL R. PRIES ${ }^{*}, 1,2$, BETTINA REGLIN ${ }^{1}$ and TIMOTHY W. SECOMB ${ }^{3}$ \\ ${ }^{1}$ Dept. of Physiology and CCR, Charité, Berlin, ${ }^{2}$ Deutsches Herzzentrum Berlin, Berlin, Germany and \\ ${ }^{3}$ Dept. of Physiology, University of Arizona, Tucson, AZ, USA
}

\begin{abstract}
Vascular beds are generated by vasculogenesis and sprouting angiogenesis, and these processes have strong stochastic components. As a result, vascular patterns exhibit significant heterogeneity with respect to the topological arrangement of the individual vessel segments and the characteristics (length, number of segments) of different arterio-venous pathways. This structural heterogeneity tends to cause heterogeneous distributions of flow and oxygen availability in tissue. However, these quantities must be maintained within tolerable ranges to allow normal tissue function. This is achieved largely through adjustment of vascular flow resistance by control of vessel diameters. While short-term diameter control by changes in vascular tone in arterioles and small arteries plays an important role, in the long term an even more important role is played by structural adaptation (angioadaptation), occurring in response to metabolic and hemodynamic signals. The effectiveness, stability and robustness of this angioadaptation depend sensitively on the nature and strength of the vascular responses involved and their interactions with the network structure. Mathematical models are helpful in understanding these complex interactions, and can be used to simulate the consequences of failures in sensing or signal transmission mechanisms. For the tumor microcirculation, this strategy of combining experimental observations with theoretical models, has led to the hypothesis that dysfunctional information transport via vascular connexins is a major cause of the observed vascular pathology and increased heterogeneity in oxygen distribution.
\end{abstract}

KEYWORDS: microvascular networks, heterogeneity, tumor, angiogenesis

\section{Introduction}

Many aspects of biological patterning and development can be investigated and, in principal, understood in terms of specific genetic and molecular mechanisms. For instance, the structure of the heart and the major arteries and veins is determined to a large degree by genetic programs. However, the microvascular system contains more than $10^{\circ}$ vessel segments, and it is obvious that the location and characteristics of each segment cannot be under individual genetic control. Instead, each vessel segment must be capable of responding appropriately to locally available information, such that multiple segments can organize themselves into functional and efficient network structures (Pries et al., 2005). In order to understand the resulting vascular structures and their responses to normal and pathological conditions, the relevant stimuli and the 'rules' governing the vascular responses should be identified. Insight into these vascular behaviors can be obtained by analyzing the structure and functional properties of normal and pathological networks, using a 'top-down' approach. In this approach, experi- mental observations are combined with theoretical simulations of blood flow and structural adaptation in vascular networks. Here, progress using this approach is reviewed, with emphasis on the inherent variability in vascular network structures and the mechanisms by which adequate functional behavior is achieved despite this heterogeneity.

\section{Heterogeneity}

A strong variability of all measured parameters, including capillary diameter, flow velocity, shear stress, perfusion and oxygen saturation is a hallmark of terminal vascular beds (Bassingthwaighte et al., 1989; Decking et al., 2001; Deussen, 1998; Duling and Damon, 1987; Pries et al., 1995b; Wagner, 2003; Zuurbier et al., 1999), even under normal physiological conditions. Increases of heterogeneity lead to anoxic regions and the possibility of tissue failure in a number of pathological conditions (Graeber et al.,

Abbreviations used in this paper: VEGF, vascular endothelial growth factor.

*Address correspondence to: Axel R. Pries. Charité Berlin, Dept. of Physiology, Thielallee 71, D-14195 Berlin, Germany. Fax: +49-30-450-528-910. e-mail: axel.pries@charite.de

ISSN: Online 1696-3547, Print 0214-6282 
1996; Marshall et al., 2003; Niimi et al., 2005; Pries et al., 2009; Secomb et al., 1993; Tyml and Mikulash, 1988). This recognition of the significance of microvascular heterogeneity is in contrast to classical approaches, in which vascular phenotypes are assumed to be identical within each of a finite number of categories, such as capillaries (Krogh, 1929; Krogh, 1919). While such approaches are useful for the analysis of some microvascular phenomena, including oxygen exchange, they cannot adequately address phenomena related to distribution of blood flow. Depending on the level of heterogeneity, the overall oxygen extraction of a given tissue can vary from a level dominated by tissue oxygen demand (at low heterogeneity) to near zero (at maximal heterogeneity), all in face of a fixed bulk perfusion of the tissue. The latter situation occurs if a few short flow pathways with very high flow rates shunt most of the blood from the feeding arteries to the draining venules. The presence of heterogeneity and the possibility of functional shunting have substantial implications for the understanding of angiogenesis and angioadaptation (Pries and Secomb, 2009). Recently we proposed that a deficiency of anti-shunt mechanisms related to information transfer along vessels (Pries et al., 2010) is central for the ineffectiveness of tumor microvasculature, leading to regions with very low oxygen availability.

\section{Angiogenesis}

During sprouting angiogenesis, new vessels are generated based on local fields of pro-angiogenic substances including VEGF

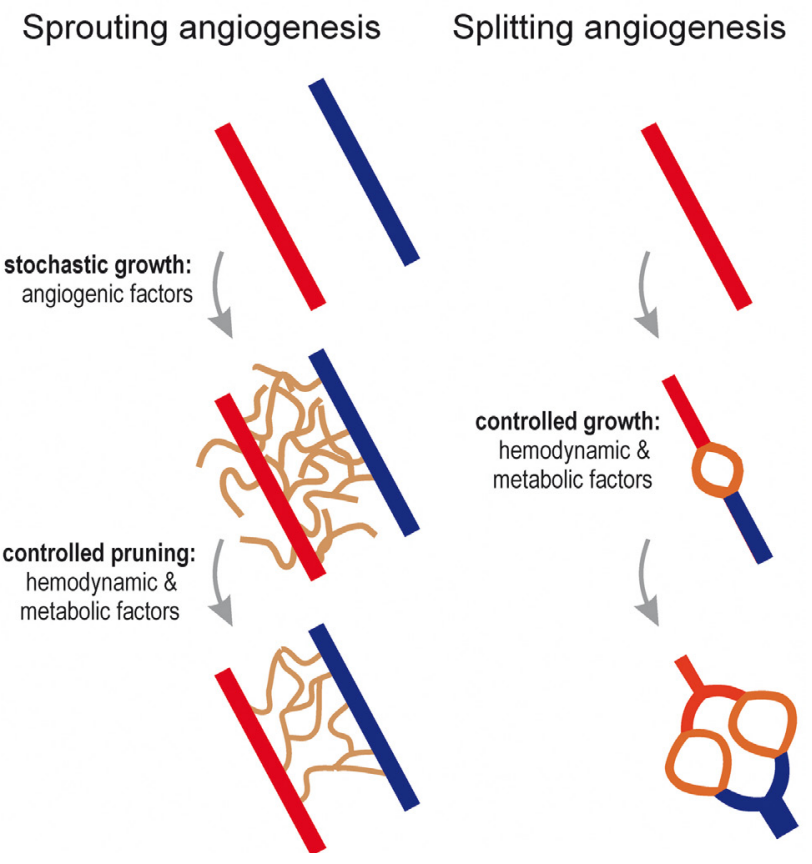

Fig. 1. Sprouting and splitting angiogenesis. During sprouting angiogenesis, local fields of pro-angiogenic factors in the tissue, for instance generated in response to hypoxia, induce stochastic growth of new vessels. The generation of a functional and efficient network requires pruning of redundant segments, governed by local hemodynamic and metabolic signals. Splitting angiogenesis (intussusception), in contrast, allows continuous feedback control by hemodynamic and metabolic conditions. Red: arterioles; brown: capillaries; blue: venules.
(Fig. 1). However, the exact locations of individual vessels are not controlled and the growing sprouts do not carry blood flow. Thus, this phase of angiogenesis occurs in the absence of any feedback with respect to the effect of the new vessels on hemodynamics and oxygen distribution. Once vessels have formed connections resulting in patent flow pathways, a second phase, pruning, is possible (Carmeliet, 2005), in which responses to hemodynamic and metabolic factors can be used to remove redundant segments, for instance those with low volume flow and high oxygen tension, and to enlarge those which are effectively supplying the tissue, which have higher flow and low distal oxygen levels. For the splitting type of angiogenesis (intussusception) (Burri, 1992; Djonov et al., 2003), the presence of continuous blood flow allows a faster adaptation of the resulting vascular pattern to tissue demand. In either case, the processes of angiogenesis and pruning, together with the need to distribute capillaries throughout all regions of the tissue, necessarily result in formation of vascular networks with long and short arterio-venous pathways (Pries and Secomb, 2009).

\section{Angioadaptation}

The vascular networks initially generated by vasculogenesis or angiogenesis (and pruning) are functionally inadequate and require adjustment of structural vessel diameters to obtain a suitable distribution of flow. Similar processes occur continuously during growth, changes in functional requirements, or as a consequence of pathophysiological states such as hypertension (Pries et al., 2005) and arteriogenesis (Schaper, 2009). Responses to hemodynamic and metabolic signals and transfer of information along vessels (Pries et al., 2001) play a central role in this remodeling process, referred to as angioadaptation (Fig. 2). The various signals and biological reactions contribute in distinct ways to the generation of functionally adequate microvascular networks. For example, the response to transmural pressure, in which increased pressure elicits wall thickening and decreased luminal diameter, leads to arteriovenous asymmetry with smaller vessels and higher flow resistance on the arterial side (Pries et al., 1995a). This asymmetry has the important consequence of reducing intracapillary pressures to a level that avoids excessive fluid filtration and control of blood flow.

The response to shear stress, in which increased shear stress leads to vessel enlargement, adjusts vessel diameters according to the flow rate within the vessel. This tends to minimize overall energy dissipation in a vascular tree (Murray, 1926). However, the feedback characteristics of this shear stress response depend on the hemodynamic boundary conditions. If the flow through a vessel is constant, the increased diameter reduces shear constituting a negative feedback which regulates shear stress levels. However, for situations where the driving pressure over a vessel is constant, the increased diameter leads to increased flow and further increases in shear, constituting a positive feedback loop. Thus for parallel flow pathways, which are subject to the same driving pressures, the response to shear stress leads to an inherent instability. The flow path with the highest shear tends to grow at the expense of all other flow pathways (Hacking et al., 1996; Pries et al., 1998; Rodbard, 1975). Here, the responses of vessel segments to metabolic stimuli play an essential role, by generating signals that tend to maintain all parallel flow pathways that are needed to maintain tissue supply (Pries et al., 1998).

Microvascular networks inevitably contain both long and short 
Fig. 2. Roles of wall shear stress, metabolic, conducted and convected signals in structural adaptation. (A,B) Local action of adaptive signals; (C,D) Modes of metabolic information transfer. Left column: mechanisms of signal generation. Center column: stimuliand responses. Dashed lines show feedback pathways (blunt heads indicate negative feedback). Right column: Significance for network properties. Flow directions are indicated by small black arrows. (A) Shear stress ( $\tau$ ) generated by flowing blood is sensed by endothelial cells and stimulates structural increase in diameter. For a given blood flow, shear stress decreases, giving a negative feedback. However, for a given driving pressure increased diameter will lead to increased flow, causing positive feedback. Shear stress response generates a progression from larger to smaller vessels correlated with flow rates in the arterial and venous trees. Transmural pressure difference leads to a circumferential wall stress ( $\sigma$ ) which in turn stimulates diameter decrease. Wall stress response generates the arterio-venous asymmetry with small arterial and larger venous vessels. (B) Tissue hypoxia leads to release of metabolic signal substances that diffuse into blood and stimulate increased diameter and increased flow, which improves oxygen availability and may also lead to faster washout of metabolic signal substance. The metabolic response enables the vasculature to respond to tissue metabolic demands and stabilizes parallel flow pathways. (C) Conducted signals generated in distal vessels are propagated upstream to feeding arterioles (orange arrow). Short arterio-venous connections (green circle) remain small and do not form functional shunts. For conduction, it is relevant that signals travel only in the direction against the blood flow (upstream) and do not reenter smaller side-branches (blunted arrow). (D) In the downstream direction, convection of metabolic substances allows for information propagation to draining vessels.

arterio-venous pathways, as discussed earlier. The short arterio-venous connections (green circle in the lower right panels of Fig. 2) tend to experience high wall shear stress, as a result of the steep pressure gradients, but otherwise experience stimuli similar to those acting on the feeding arterioles from which they branch. They would tend to enlarge, forming functional arterio-venous shunts, if they were subject only to the stimuli responses discussed so far. Suppression of shunt formation requires propagation to each segment of information about the extent and metabolic status of the segments distal to that segment (Pries et al., 1998). The mechanisms of this information transfer are not fully established. However, it is likely that downstream information transfer depends on the convective transport of metabolic signaling substances, while propagation in the upstream direction is provided primarily by conducted responses that travel along the walls of microvessels via gap junctions (Pries et al., 2003; Pries et al., 2010).

As discussed below ('Tumor microcirculation'), impair-

Fig. 3. Methods for simulation of structural diameter adaptation. Experimental data on network structure and microvascular rheology serve as the basis to calculate blood flow and oxygen transport in networks with a given angioarchitecture. Stimuli derived from hemodynamic and metabolic conditions are used to predict diameter adaptation for each vessel according to a set of general adaptation rules to be tested. The properties of the vascular network after simulated adaptation are then compared with experimental network data (segment flow velocity, hematocrit and diameter) to assess the adequacy of the adaptation rules used and to optimize adaptation parameters.
$A$

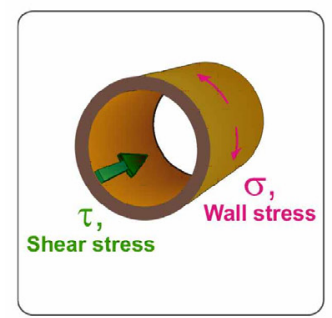

B

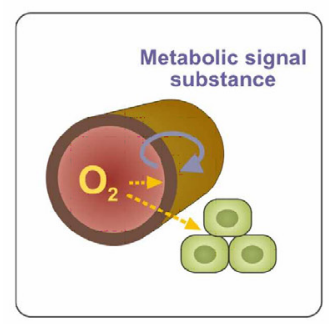

C

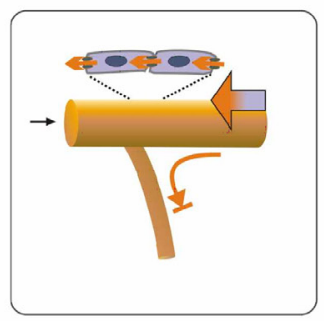

D

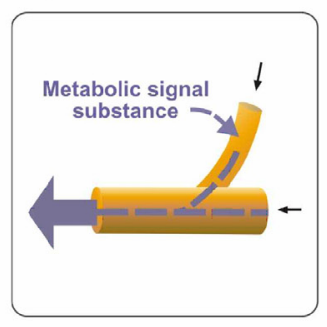

Hemodynamic signals

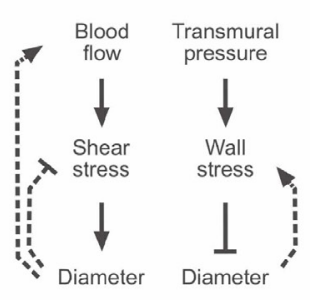

\section{Metabolic signals}
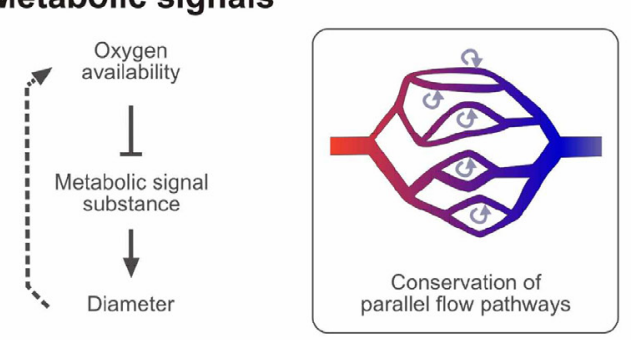

Conducted signals

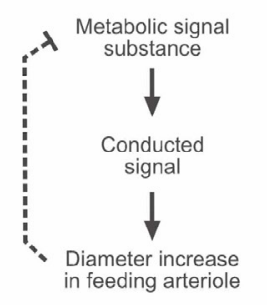

\section{Convected signals}

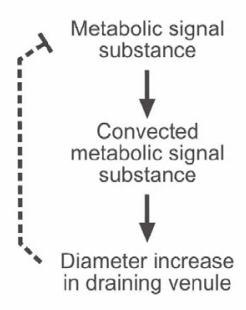

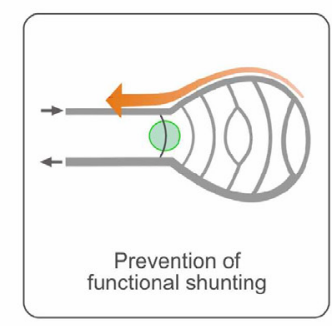
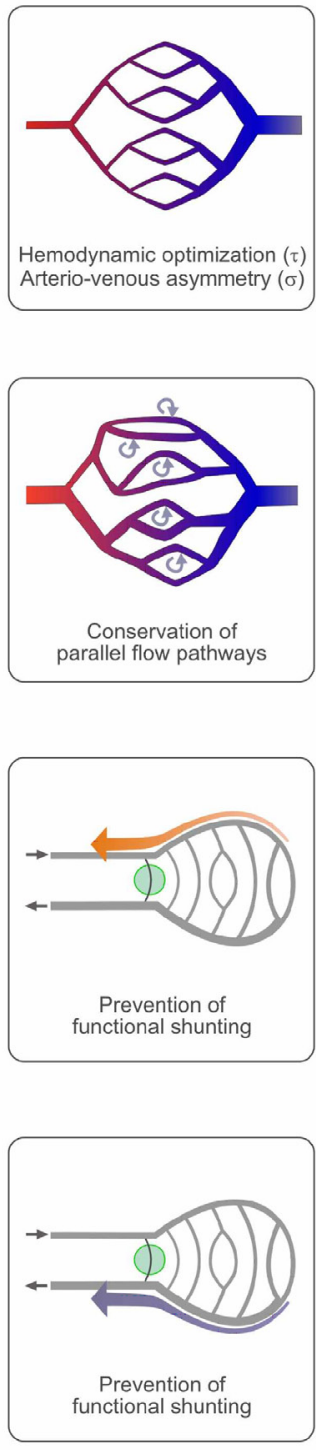

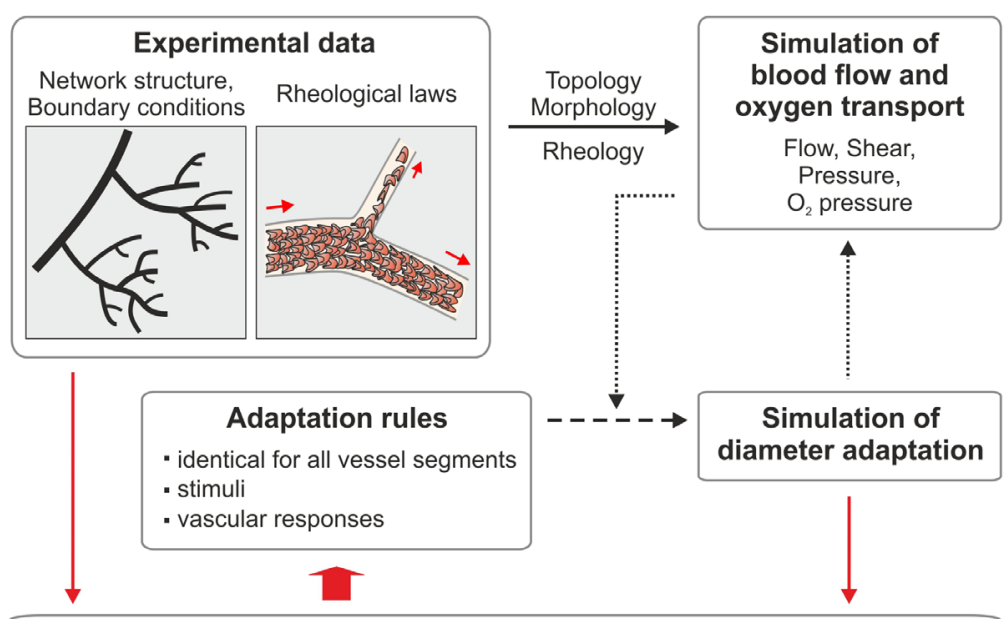

Optimization of adaptation rules

by minimizing deviation between measured and estimated parameters (e.g. flow velocity) 
ment of shunt preventing mechanisms, most importantly information transfer by conducted responses traveling via vascular gap junctions, can lead to significant dysfunction and maldistribution in vascular networks. The importance of gap junctions for angioadaptation was recently supported by findings in developing vascular beds (Buschmann et al., 2010). It was shown that gap junction proteins (Cx 40, Gja5) are expressed throughout growing arterial vessel trees from the very onset of the development of hierarchical vascular structures. It is therefore very likely that these proteins are not only 'markers' but 'makers' of the observed vascular patterns by virtue of their involvement in information transfer.

\section{Experimental data and model simulations}

Acentral approach in the analysis of the balance and interaction of angioadaptive stimuli, biological reactions and functional consequences is the combination of experimental data with computer simulations (Fig. 3) (Secomb et al., 2008). Intravital microscopy was used to determine the angioarchitecture (vessel segment diameters and length, segment connection patterns) for complete microvascular networks. In addition, measurements of flow velocity and hematocrit in all vessel segments were performed (Pries et al., 1990). The simulations are also based on experimental data concerning the microrheological behavior of blood flowing through

A

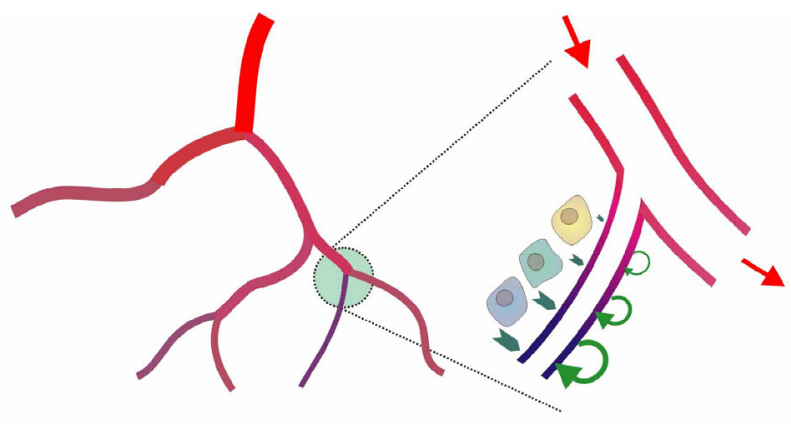

B
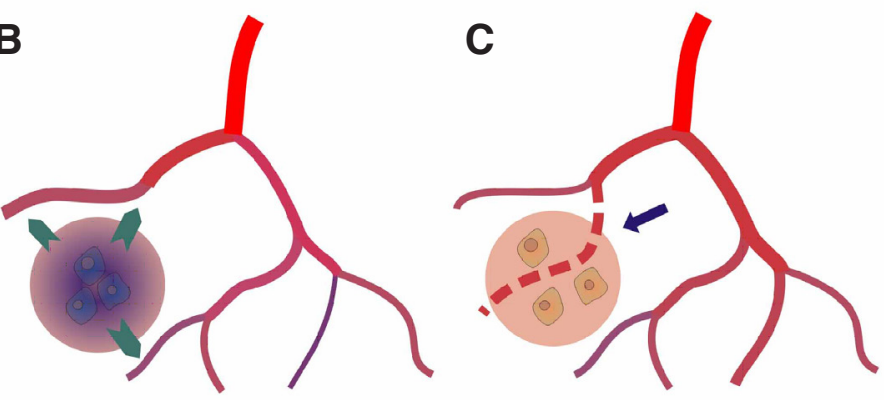

Fig. 4. Responses to oxygen supply-demand mismatch. (A) Left: Network containing a vessel with low perfusion resulting in hypoxia (blue color). Right: Enlarged view of hypoxic region. Signals generated in the vessel wall or the parenchymal tissue (green arrows) increase in proportion to local oxygen deficiency and stimulate diameter increase. This increase increases perfusion and oxygen availability constituting a negative feedback between tissue hypoxia and metabolic signaling. (B) If vessel density is too low, diameter increases lead to increased blood flow but oxygen supply of remote tissue cells remains inadequate. Supply of remote tissue cells requires stimulation of angiogenesis by tissue derived metabolic signals. (C) New vessel generated by sprouting angiogenesis (dashed line) results in reduction of diffusion distances and adequate tissue oxygenation. microvessels (Pries et al., 1992; Pries et al., 1994) and microvascular bifurcations (Pries et al., 1989). Parametric representations of these data describe the Fahraeus effect and Fahraeus-Lindqvist effect as well as the partitioning of hematocrit at diverging microvascular branch points (Pries and Secomb, 2008).

From the data on microvascular angioarchitecture and rheology, the distribution of blood flow, pressure and oxygen within a given network are calculated (Pries et al., 1990; Pries et al., 1995b). The information on shear stress, transmural pressure and oxygen partial pressure for each vessel segment is then the basis for estimating the stimuli that evoke adaptive changes and the resulting structural changes, such as diameter increase or decrease (Pries et al., 1998; Pries et al., 2005). In this process, different sets of 'adaptation rules' describing the vascular responses to a given set of adaptive stimuli and modes of information transfer can be tested. The validity of a given set of rules is then tested by comparing the distribution of vessel diameters, hematocrits and flow velocities with the corresponding values measured by intravital microscopy.

\section{Metabolic signals: structural diameter regulation and sprouting}

It is reasonable to assume that the maintenance of an adequate oxygen supply to tissues occurs largely through vascular responses to stimuli that depend on the metabolic status of tissue, including local oxygen levels (Reglin et al., 2009). However, the specific mechanisms involved have not been fully identified. Here, a theoretical approach is helpful in that it can be used to predict the functional consequences and effectiveness of different types of mechanisms. As shown in Fig. 4 (upper panel), metabolic signals generated in the vessel wall or the parenchymal tissue increase in proportion to a demand-supply mismatch and can lead to diameter increase. This increase, in turn, increases blood supply and oxygen delivery. A detailed analysis showed that the match between experimental and simulated results was best assuming that the metabolic sensor is located in the vessel wall (Reglin et al., 2009). However, this feedback mechanism cannot be effective if the vessel density is too low and diffusion distances are too high (Fig. 4, lower panels). In that case, signals originating in hypoxic tissue regions, such as diffusible vascular growth factors, must be capable of stimulating the growth of new blood vessels by (sprouting) angiogenesis (Carmeliet, 2005).

\section{Tumor microcirculation}

An example of the changes in functional characteristics of vascular networks resulting from faulty vascular adaptation is provided by the tumor microcirculation (Pries et al., 2009; Sorg et al., 2008). The heterogeneous oxygen distribution, including hypoxic and anoxic regions, is a serious problem for radio- or chemotherapy (Hicks et al., 2006; Moeller et al., 2007). Based on studies using the approach described here and observations of microvascular network structure in tumors, we recently concluded that a defect in the conducted responses along vessel walls is the main cause for the observed aberrant structural and functional properties (Pries et al., 2009). The resulting reduction of upstream information transfer along vessels leads according to the reasoning presented above to the formation of functional arterio-venous shunts and inefficient distribution of flow and oxygen. This result was obtained irrespective 

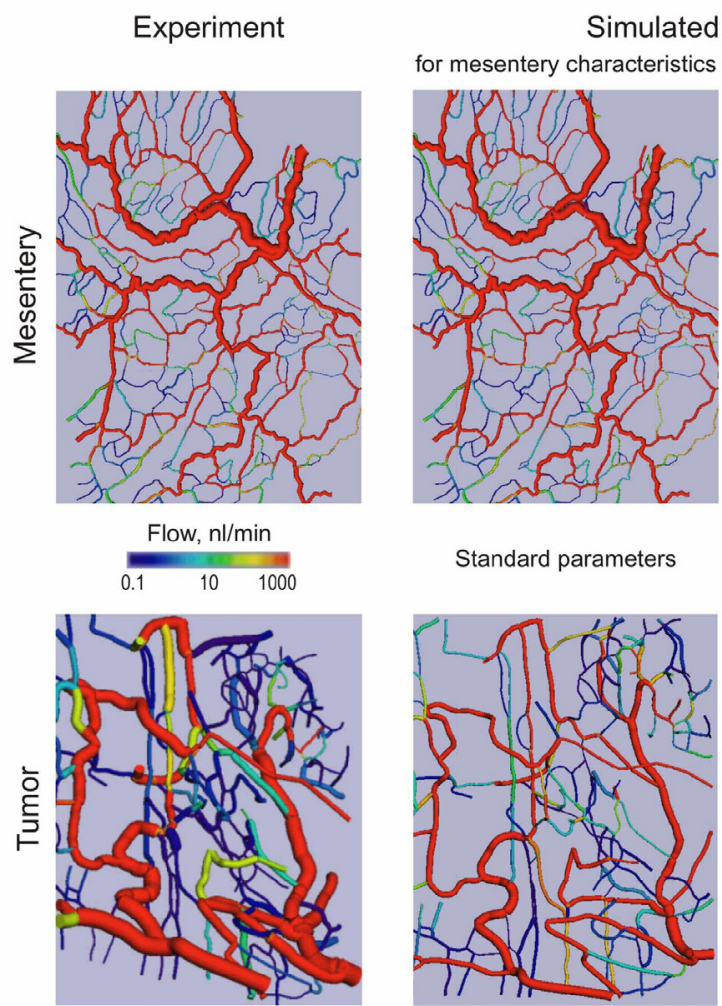

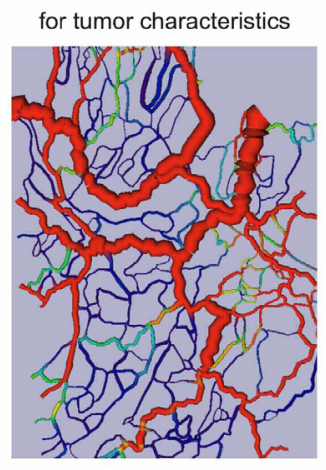

Conduction Randomness Growth stimulus
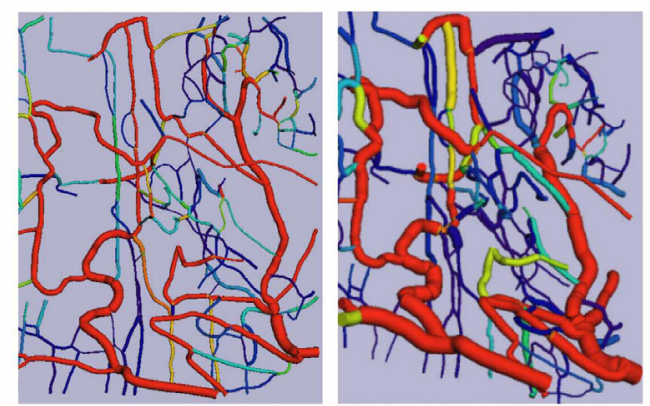

Fig. 5. Faulty vascular adaptation as a cause for aberrant tumor microcirculatory properties. Vascular patterns generated by using normal and modified ('tumor') adaptation rules in a mesenteric network (upper row) and a tumor network (lower row) are compared to results obtained with experimentally measured vessel diameters. Experimental data are shown in the left column. Simulated 'normal adaptation' (middle column), using standard model parameters optimized for normal network characteristics leads to adequate blood flow distribution in both the normal and the tumor network. In contrast, simulated 'tumor adaptation' (right column), with abolished information transfer via conduction results in increased structural heterogeneity, functional shunting and large underperfused areas, in comparison to the mesenteric network.

concepts and models for understanding the interplay and coordination of these processes. Responses to multiple stimuli may be involved in each aspect of the system. For an example, consider the short and long-term responses of vascular networks to inadequate oxygen supply. The arguments given above imply that signals derived from the vessel wall are most suitable to drive structural vascular diameter adaptation (Reglin et al., 2009). In contrast, the considerations detailed in Fig. 4 suggest that in situations with excessive diffusion distances where the generation of new vessels by sprouting angiogenesis is required, tissue-derived factors are involved. Finally, factors derived from red blood cells have been shown to be important in

of the specific network topology considered. For example, normal adaptation characteristics were shown to generate network properties very close to those measured in normal networks irrespective even when a tumor network topology was used as a starting point (Fig. 5) (Pries et al., 2009). In contrast, vascular adaptation without conducted responses led to functional shunting and large underperfused areas, even when a normal vascular network structure was used as the initial condition.

\section{Integration of vascular responses}

As indicated by the preceding discussion, the control of blood flow distribution in tissues depends on a large number of biological processes, including angiogenesis, pruning, structural adaptation of vessel diameter and wall thickness, and changes in vascular tone (Fig. 6). A major remaining challenge is the development of the control of vascular tone (Arciero et al., 2008; Ellsworth et al., 1995; Ellsworth et al., 2009).

Splitting (intussusception) and sprouting are two distinct modes of angiogenesis. A combination of metabolic and hemodynamic factors may determine which of these two modes occurs. The differentiation appears to be governed by specific patterns of oxygen availability, angiogenic factors and hemodynamic conditions (Djonov et al., 2003; Hudlicka et al., 2006; Hudlicka and Brown, 2009; Makanya et al., 2005; Milkiewicz et al., 2001). Generally, higher levels of shear stress and oxygen partial pressure seem to favor splitting angiogenesis, while sprouting is more stimulated by angiogenic factors released from the tissue in response to hypoxia.

Aclose relationship exists between the slower changes of vessel diameter by structural adaptation and the fast regulation by changes in smooth muscle tone (Carlson et al., 2008), occurring for instance in response to changes in intravascular pressure. It was shown in

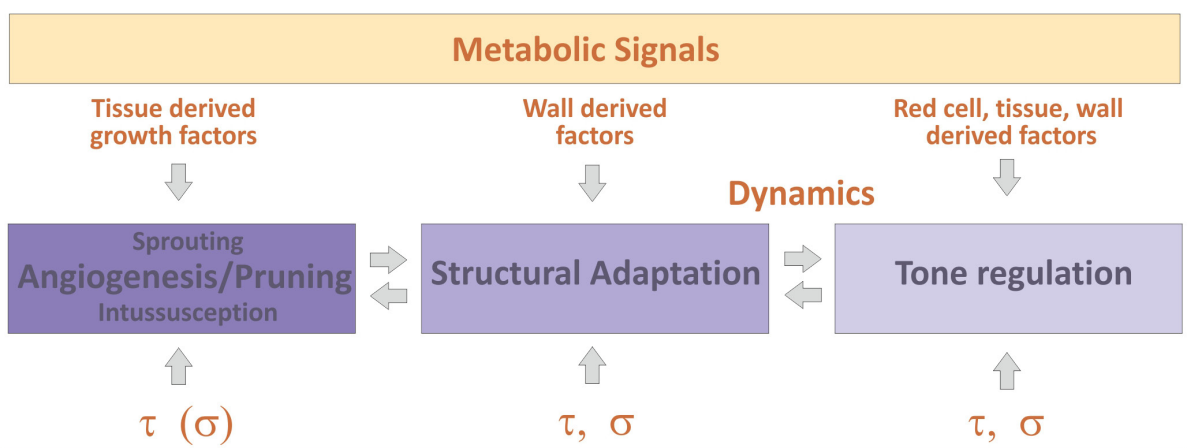

Hemodynamic Signals

$\begin{array}{cc}\text { slow (days) } & \text { slow (hours/ days) } \\ \text { high threshold } & \text { low threshold }\end{array}$

low threshold fast (seconds) low threshold
Fig. 6. Integrated view of physiological processes controlling vascular structure and function. Vascular regulation includes short term changes of vessel diameter effected by vascular smooth muscle and tone (right), long term changes of vessel diameter and wall mass by structural adaptation (middle), and changes in vessel number by elimination of unnecessary vessels by pruning or generation of new vessels by the sprouting and splitting modes of angiogenesis (left). These responses of a vascular bed are controlled by metabolic and hemodynamic signals derived, e.g., from the tissue, the vessel wall or red cells, and by hemodynamic signals (wall shear stress, $\tau$; wall stress, $\sigma$ ). The different modes of vascular regulation are closely interrelated and may be induced by different combinations of the involved stimuliand by specific temporal and dynamic patterns of these stimuli (see text for further explanations). 
experiments with isolated arterioles that sustained vasoconstriction leads to inward remodeling (Bakker et al., 2002). Also, structural diameter adaptation leading to a continuous decrease of vessel diameter may ultimately lead to the removal of the vessel segment by pruning. The various modes of vascular regulation described in this review may thus be elicited by different specific combinations of stimuli and by particular spatial and temporal patterns. More study of these processes and their interactions is needed to better understand the control of vascular structure and perfusion and to support the development of corresponding therapeutic strategies.

\section{References}

ARCIERO JC, CARLSON BE and SECOMB TW (2008). Theoretical model of metabolic blood flow regulation: Roles of ATP release by red blood cells and conducted responses. Am J Physiol Heart Circ Physiol 295: H1562-H1571.

BAKKER EN, VAN DER MEULEN ET, VAN DEN BERG BM, EVERTS V, SPAAN JA and VANBAVEL E (2002). Inward remodeling follows chronic vasoconstriction in isolated resistance arteries. J Vasc Res 39: 12-20.

BASSINGTHWAIGHTE JB, KING RB and ROGER SA (1989). Fractal nature of regional myocardial blood flow heterogeneity. Circ Res 65: 578-590.

BURRI PH (1992). Intussusceptive microvascular growth, a new mechanism of capillary network formation. EXS 61: 32-39.

BUSCHMANN I, PRIES A, STYP-REKOWSKA B, HILLMEISTER P, LOUFRANI L, HENRION D, SHIY, DUELSNERA, HOEFER I, GATZKE N, WANG H, LEHMANN K, ULM L, RITTER Z, HAUFF P, HLUSHCHUK R, DJONOV V, VAN VEEN T and LE NOBLE F. (2010). Pulsatile shear and Gja5 modulate arterial identity and remodeling events during flow-driven arteriogenesis. Development 137:2187-2196.

CARLSON BE, ARCIERO JC and SECOMB TW (2008). Theoretical model of blood flow autoregulation: Roles of myogenic, shear-dependent and metabolic responses. Am J Physiol Heart Circ Physiol. 295: H1572-H1579.

CARMELIETP (2005). Angiogenesis in life, disease and medicine. Nature 438: 932-936.

DECKING UK, SKWIRBA S, ZIMMERMANN MF, PRECKEL B, THAMER V, DEUSSEN A and SCHRADER J (2001). Spatial heterogeneity of energy turnover in the heart. Pflugers Arch 441: 663-673.

DEUSSEN A (1998). Blood flow heterogeneity in the heart. Basic Res Cardiol 93: 430-438.

DJONOV V, BAUM O and BURRIPH (2003). Vascular remodeling by intussusceptive angiogenesis. Cell Tissue Res 314: 107-117.

DULING BR and DAMON DH (1987). An examination of the measurement of flow heterogeneity in striated muscle. Circ Res 60: 1-13.

ELLSWORTH ML, ELLIS CG, GOLDMAND, STEPHENSON AH, DIETRICH HH and SPRAGUE RS (2009). Erythrocytes: oxygen sensors and modulators of vascular tone. Physiology (Bethesda) 24: 107-116.

ELLSWORTH ML, FORRESTER T, ELLIS CG and DIETRICH HH (1995). The erythrocyte as a regulator of vascular tone. Am J Physiol 269: $\mathrm{H} 2155-\mathrm{H} 2161$.

GRAEBER TG, OSMANIAN C, JACKS T, HOUSMAN DE, KOCH CJ, LOWE SW and GIACCIA AJ (1996). Hypoxia-mediated selection of cells with diminished apoptotic potential in solid tumours. Nature 379: 88-91.

HACKING WJG, VANBAVELE and SPAAN JAE (1996). Shear stress is not sufficient to control growth of vascular networks: a model study. Am J Physiol270: H364-H375.

HICKS KO, PRUIJN FB, SECOMB TW, HAY MP, HSU R, BROWN JM, DENNY WA, DEWHIRSTMW and WILSONWR(2006). Use of three-dimensional tissue cultures to model extravascular transport and predict in vivo activity of hypoxia-targeted anticancer drugs. J Natl Cancer Inst 98: 1118-1128.

HUDLICKA O and BROWN MD (2009). Adaptation of skeletal muscle microvasculature to increased or decreased blood flow: role of shear stress, nitric oxide and vascular endothelial growth factor. J Vasc Res 46: 504-512.

HUDLICKAO, BROWN MD, MAYS, ZAKRZEWICZA and PRIESAR (2006). Changes in capillary shear stress in skeletal muscles exposed to long-term activity: role of nitric oxide. Microcirculation 13: 249-259.

KROGH A (1919). The supply of oxygen to the tissues and the regulation of the capillary circulation. J Physiol (Lond) 52: 457-474.

KROGHA (1929). The Anatomy and Physiology of Capillaries. Yale University Press,
New Haven, CT.

MAKANYA AN, STAUFFER D, RIBATTI D, BURRI PH and DJONOV V (2005). Microvascular growth, development, and remodeling in the embryonic avian kidney: the interplay between sprouting and intussusceptive angiogenic mechanisms. Microsc Res Tech 66: 275-288.

MARSHALL RC, POWERS-RISIUS P, REUTTER BW, SCHUSTZ AM, KUO C, HUESMAN MK and HUESMAN RH (2003). Flow heterogeneity following global no-flow ischemia in isolated rabbit heart. Am J Physiol 284: $\mathrm{H} 654-\mathrm{H} 667$.

MILKIEWICZ M, BROWN MD, EGGINTON S and HUDLICKA O (2001). Association between shear stress, angiogenesis, and VEGF in skeletal muscles in vivo. Microcirculation 8: 1-13.

MOELLER BJ, RICHARDSON RA and DEWHIRST MW (2007). Hypoxia and radiotherapy: opportunities for improved outcomes in cancer treatment. Cancer Metastasis Rev 26: 241-248.

MURRAY CD (1926). The physiological principle of minimum work. I.The vascular system and the cost of blood volume. Proc Natl Acad Sci USA 12: 207-214.

NIIMI H, NAKANO A, KOMAI Y and SEKI J (2005). Heterogeneity of capillary flow in the retrograde microcirculation induced in rat limb by arteriovenous shunting. Microvasc Res 70: 23-31.

PRIES AR, CORNELISSEN AJ, SLOOT AA, HINKELDEY M, DREHER MR, HOPFNER M, DEWHIRST MW and SECOMB TW (2009). Structural adaptation and heterogeneity of normal and tumor microvascular networks. PLoS Comput Biol 5: e1000394.

PRIES AR, HOPFNER M, LE NOBLE F., DEWHIRST MW and SECOMB TW (2010). The shunt problem: control of functional shunting in normal and tumour vasculature. Nat Rev Cancer 10: 587-593.

PRIES AR, LEY K, CLAASSEN M and GAEHTGENS P (1989). Red cell distribution at microvascular bifurcations. Microvasc Res 38: 81-101.

PRIES AR, NEUHAUS D and GAEHTGENS P (1992). Blood viscosity in tube flow: dependence on diameter and hematocrit. Am J Physiol 263: $\mathrm{H} 1770-\mathrm{H} 1778$.

PRIESAR, REGLINB and SECOMBTW (2001). Structural adaptation of microvascular networks: functional roles of adaptive responses. Am J Physiol281: $\mathrm{H} 1015-\mathrm{H} 1025$.

PRIES AR, REGLIN B and SECOMB TW (2003). Structural response of microcirculatory networks to changes in demand: information transfer by shear stress. $\mathrm{Am}$ J Physiol 284: $\mathrm{H} 2204-\mathrm{H} 2212$.

PRIES AR, REGLIN B and SECOMB TW (2005). Remodeling of blood vessels: responses of diameter and wall thickness to hemodynamic and metabolic stimuli. Hypertension 46: 726-731.

PRIES, A.R. AND SECOMB, T.W. (2008). Blood flow in microvascular networks. In Handbook of Physiology: Microcirculation (Eds. R.F. Tuma, W.N. Durán and K. Ley). Academic Press, Elsevier, San Diego, pp. 3-36.

PRIES AR and SECOMB TW (2009). Origins of heterogeneity in tissue perfusion and metabolism. Cardiovasc Res 81: 328-335.

PRIES AR, SECOMB TW and GAEHTGENS P (1995b). Structure and hemodynamics of microvascular networks: heterogeneity and correlations. Am J Physiol 269: $\mathrm{H} 1713-\mathrm{H} 1722$.

PRIESAR, SECOMB TW and GAEHTGENS P (1995a). Design principles of vascular beds. Circ Res 77: 1017-1023.

PRIES AR, SECOMB TW and GAEHTGENS P (1998). Structural adaptation and stability of microvascular networks: theory and simulations. Am J Physiol 275: H349-H360.

PRIES AR, SECOMB TW, GAEHTGENS P and GROSS JF (1990). Blood flow in microvascular networks - experiments and simulation. Circ Res 67: 826-834.

PRIES AR, SECOMB TW, GESSNER T, SPERANDIO MB, GROSS JF and GAEHTGENS P (1994). Resistance to blood flow in microvessels in vivo. Circ Res 75: $904-915$

REGLIN B, SECOMB TW and PRIESAR (2009). Structural adaptation of microvessel diameters in response to metabolic stimuli: where are the oxygen sensors? Am $J$ Physiol Heart Circ Physiol 297: H2206-H2219.

RODBARD S (1975). Vascular caliber. Cardiology 60: 4-49.

SCHAPERW (2009). Collateral circulation: pastand present. Basic Res Cardio/104:5-21.

SECOMB TW, BEARD DA, FRISBEE JC, SMITH NP and PRIES AR (2008). The role of theoretical modeling in microcirculation research. Microcirculation 15:693-698.

SECOMB TW, HSU R, DEWHIRST MW, KLITZMAN B and GROSS JF (1993). Analysis of oxygen transport to tumor tissue by microvascular networks. Int $J$ 
Radiat Oncol Biol Phys 25: 481-489.

SORG BS, HARDEE ME, AGARWAL N, MOELLER BJ and DEWHIRST MW (2008). Spectral imaging facilitates visualization and measurements of unstable and abnormal microvascular oxygen transport in tumors. J Biomed Opt 13: 014026.

TYML K and MIKULASH K (1988). Evidence for increased perfusion heterogeneity in skeletal muscle during reduced flow. Microvasc Res 35: 316-324.

WAGNER PD (2003). Heterogeneity of skeletal muscle perfusion and metabolism. J Appl Physiol 95: 2202-2203.

ZUURBIER CJ, VAN ITERSON M and INCE C (1999). Functional heterogeneity of oxygen supply-consumption ratio in the heart. Cardiovasc Res 44: 488-497.

\section{Further Related Reading, published previously in the Int. J. Dev. Biol.}

Development of a fast, objective, quantitative methodology to monitor angiogenesis in the chicken chorioallantoic membrane during development

Eva Verhoelst, Bart De Ketelaere, Veerle Bruggeman, Eduardo Villamor, Eddy Decuypere and Josse De Baerdemaeker

Int. J. Dev. Biol. (2011) 55: 85-92

The role of pericytes in angiogenesis

Domenico Ribatti, Beatrice Nico and Enrico Crivellato

Int. J. Dev. Biol. (2011) 55: 261-268

The seminal work of Werner Risau in the study of the development of the vascular system

Domenico Ribatti

Int. J. Dev. Biol. (2010) 54: 567-572

Shear stress regulation of nitric oxide production in uterine and placental artery endothelial cells: experimental studies and hemodynamic models of shear stresses on endothelial cells

Benjamin Sprague, Naomi C. Chesler and Ronald R. Magness

Int. J. Dev. Biol. (2010) 54: 331-339

Microarchitectural changes during development of the cerebellar cortex

Miriam Mecha, Angel L. Peña-Melián and Maria J. Blanco

Int. J. Dev. Biol. (2010) 54: 691-698

XRASGRP2 is essential for blood vessel formation during Xenopus development

Kan Suzuki, Shuji Takahashi, Yoshikazu Haramoto, Yasuko Onuma, Kentaro Nagamine, Koji Okabayashi, Kohei Hashizume, Tadashi Iwanaka and Makoto Asashima

Int. J. Dev. Biol. (2010) 54: 609-615

Insulin-like growth factor-2 regulates early neural and cardiovascular system development in zebrafish embryos Lori Hartnett, Catherine Glynn, Catherine M. Nolan, Maura Grealy and Lucy Byrnes

Int. J. Dev. Biol. (2010) 54: 573-583

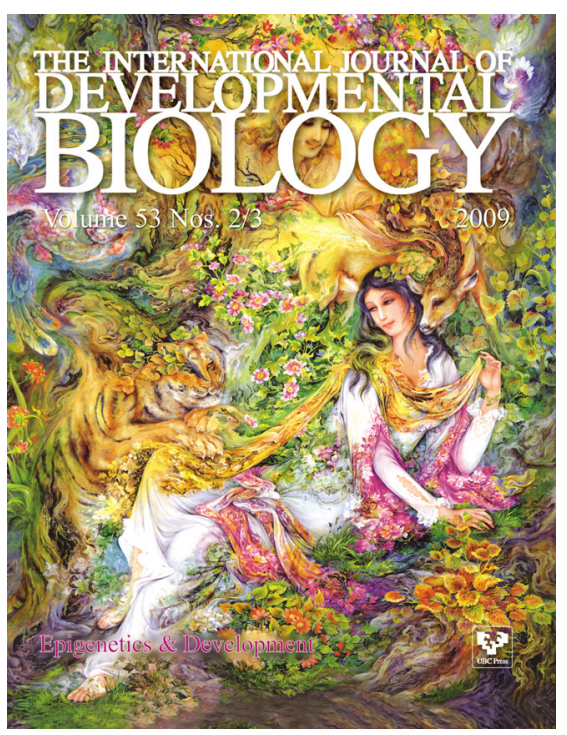

5 yr ISI Impact Factor $(2009)=3.253$

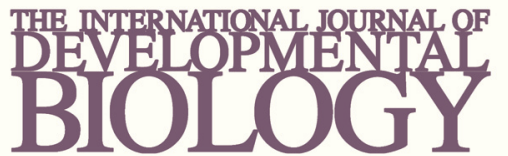

Volume 54 Nos. 6/7

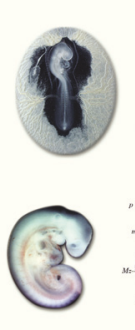

Developmental Hematopoiesis
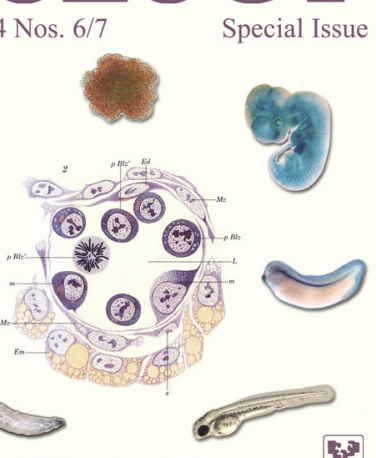

\$

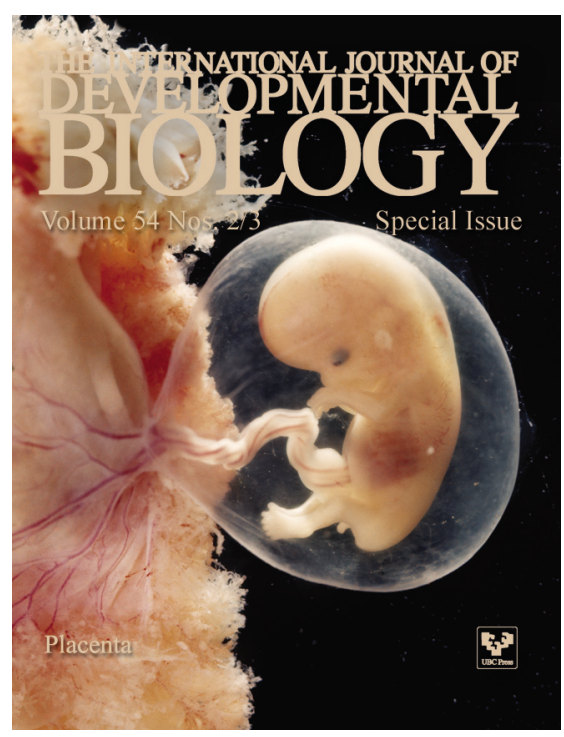

